\title{
Pre-operative corticosteroid injections improve functional outcomes in patients undergoing arthroscopic repair of high-grade partial-thickness rotator cuff tears
}

\author{
Nicholas K. Donohue ${ }^{1}$ \\ Anthony R. Prisco ${ }^{2}$ \\ Steven I. Grindel ${ }^{1}$ \\ 1 Department of Orthopaedic Surgery, Medical \\ College of Wisconsin, Milwaukee, USA \\ 2 Department of Medicine,University of Minnesota, \\ Minneapolis, USA
}

Corresponding author:

Nicholas Donohue

Department of Orthopaedic Surgery,

Medical College of Wisconsin

9200 West Wisconsin Ave, 53226 Milwaukee, USA

E-mail: nkdonohue@mcw.edu

\section{Summary}

Background: Subacromial corticosteroid injections (CSI's) are a common non-surgical treatment for rotator cuff tears. Few studies have assessed the effects of pre-operative CSI's on postoperative functional outcomes.

Methods: A retrospective analysis was conducted of 132 patients with high-grade, partial-thickness rotator cuff tears (PTRCT's). The subjects were divided into two groups based on whether they received a CSI or not. The CSI group was further divided into three subgroups based on when they received a pre-operative injection: 0-3 months, 3-6 months, $>6$ months before surgery. The Visual Analog Scores (VAS), American Shoulder and EIbow Surgeon scores (ASES), and Constant scores were recorded prior to surgery and at a one-year post-operative follow-up appointment for each subject.

Results: Patients who received a pre-operative CSI $(n=92)$ improved significantly more than the non-injection group $(n=40)$ in all outcome measures. The 0-3 months injection subgroup experienced a significant increase in ASES and Constant score ( $p=0.019$ and 0.014 , respectively) compared to the other two subgroups, but the VAS score decrease only trended toward significance $(p=0.091)$. The sample as a whole experienced significant improvement in all three outcome measures.

Conclusion: Patients undergoing arthroscopic re- pair of a high-grade PTRCT may benefit from a pre-operative CSI 0-3 months before surgery. Level of evidence: Ilb.

KEY WORDS: partial-thickness rotator cuff tear, rotator cuff, subacromial injection, corticosteroid injection, arthroscopic repair.

\section{Introduction}

A rotator cuff tear (RCT) is a common and painful problem for approximately $20 \%$ of the general population that increases in occurrence with age ${ }^{1}$. The most common RCT involves the supraspinatus tendon, and these tears can be divided into partial-thickness rotator cuff tears (PTRCT's) or full-thickness tears. PTRCT's can be further subdivided into highgrade or low-grade based on whether a majority of the tendon's insertion into the greater tuberosity is involved ${ }^{2}$.

Subacromial corticosteroid injections (CSI's) are a common non-surgical treatment for rotator cuff tears (RCT's) and other shoulder pathologies. While the science behind the use of CSI's is currently inconclusive ${ }^{3-10}$, they are typically employed along with other non-surgical measures like physical therapy, activity modification, and non-steroidal anti-inflammatory medications (NSAIDs), in order to reduce pain and increase functionality in patients with RCT's. When a combination of these therapies does not relieve patients' symptoms sufficiently, surgical repair is typically the next treatment. Thus, the role of subacromial CSI's in current RCT treatment protocols is to provide symptom relief in lieu of invasive surgery. Their use as a pre- or perioperative anti-inflammatory agent in conjunction with surgery to maximize post-operative results is novel.

To our knowledge, no study has investigated whether pre-operative CSI's have any impact on post-operative functional outcomes. Theoretically, if administered close enough to surgery the anti-inflammatory effects of the corticosteroid could provide increased pain relief to allow for earlier and increased post-operative rehabilitation. The concept of administering peri-operative local anesthetic injections in order to improve post-operative results is well-documented in hip and knee surgery ${ }^{11-17}$ but to our knowledge nothing has been written on a similar approach to RCT repair.

The purpose of this study was to examine whether 
pre-operative CSI's have an impact on post-operative functional outcomes following arthroscopic repair of PTRCT's, and if so, whether the timing of the injection plays an important role. We hypothesize that administration of a subacromial CSI 0-3 months prior to surgery will result in significantly better outcomes one year after surgery.

\section{Materials and methods}

This study obtained approval from the Institutional Review Board of our institution and was conducted in accord with international standards and the ethical standards of Muscle, Ligaments and Tendon Journall8. A retrospective review was performed on the charts of patients who underwent arthroscopic repair of a high-grade partial-thickness tear of the supraspinatus tendon between 2008 and 2014. Patients were included in the study if they successfully completed all questionnaires prior to surgery and at a follow-up appointment one year after repair. A pre-operative diagnosis of the PTRCT was based on a combination of patient history, physical exam, and magnetic resonance imaging (MRI). MRI findings were then compared to intra-operative findings to confirm tear size and location. All procedures were performed by a single, board-certified orthopaedic surgeon. Patients who had prior surgery on the ipsilateral shoulder, a low-grade PTRCT of the supraspinatus tendon, or a tear which extended into other rotator cuff tendons were excluded from the study. Patients who experienced trauma to the affected shoulder within one year after surgery were also excluded from the study. Patients were divided into groups based on whether they received a pre-operative CSI or not. The CSI group was then further divided into three CSI subgroups: 1) patients who received a CSI 0-3 months prior to surgery, 2) those who received a CSI 3-6 months prior, and 3 ) a group who received a $\mathrm{CSI}>6$ months before surgery. The CSI itself consisted of 4 cc of $1 \%$ lidocaine without epinephrine and $6 \mathrm{mg}$ betamethasone administered to the subacromial space. Diagnostic arthroscopy was first performed to assess the rotator cuff. Tear depth and percentage was determined with the aid of a calibrated arthroscopic probe with a $3 \mathrm{~mm}$ bent arm once debridement of degenerative tissue was completed. All PTRCT's were then converted to a full-thickness tear, and the rotator cuff footprint on the greater tuberosity was debrided to bleeding cortical bone. Depending on tear length, one or two Arthrex Bio-Corkscrew absorbable suture anchors with two \# 2 Fiberwire sutures (Arthrex Inc., Naples, FL) were then placed in the tuberosity 3-5 $\mathrm{mm}$ lateral to the articular margin. The sutures were passed through the rotator cuff with an arthroscopic suture passer such that one suture was placed in horizontal mattress and the other was placed in a simple fashion deep to the horizontal mattress. The sutures were then tied with a modified Roeder knot. Concomitant procedures were included in the study and limited to subacromial decompression, acromioclavicular resection, superior labrum anterior-posterior debridement, and biceps tenotomy or tenodesis.

Post-operatively, a standardized rehabilitation protocol was initiated by all patients starting within the first week of surgery. Passive range of motion (ROM) was performed for the first 6 weeks, followed by active ROM from 6 to 8 weeks after surgery. Strengthening was then initiated at 8 weeks following surgery.

Pre- and post-operative assessment was made by the use of three functional outcome measurements: Visual Analog Scale (VAS), American Shoulder and Elbow Surgeons score (ASES), and the ConstantMurley score (CMS). Patients were asked at their initial appointment to fill out standard questionnaires and were examined by the surgeon. The post-operative data were gathered 12 months after surgery during the end-of-healing exam for all patients.

A pre- and post-intervention evaluation of patient function was conducted on each patient using three metrics: a VAS score, ASES score, and a Constant score. Statistical analyses were conducted on the magnitude of the change between the pre- and posttest scores. A paired student's t-test was used to evaluate changes in response to the intervention(s) in each metric. An ANOVA was initially used to compare the change in mean scores between the three injection subgroups. Once significance was established, paired t-tests were then used to compare results between injection subgroups. A two-way ANOVA was used to compare the effectiveness of cortisone injection and physical therapy on each metric. Finally, a multiple linear regression analysis was run for each metric in order to determine the temporal relationship of cortisone injection to functional improvement. In these analyses, potential confounders were analyzed as well, which included age, gender, tear location (articular, bursal, or intratendinous), pre-operative physical therapy (yes or no), concomitant procedures, tear etiology (traumatic $v s$ insidious) and duration of symptoms prior to surgery (0-3 months, 3-6 months, and $>6$ months prior to surgery). A p-value $<0.05$ was considered statistically significant.

\section{Results}

A total of 132 patients was included in this retrospective review. A summary of the patient demographics is provided in Table I. The average patient age was 48.2 years (range 29-77), and the gender division was 62 females to 70 males. Concomitant procedures were limited to subacromial decompression (92\%), acromioclavicular resection $(67 \%)$, superior labrum anterior-posterior debridement $(37 \%)$, and biceps tenotomy/tenodesis (19\%). The sample as a whole experienced significant improvement in all three outcome measures: VAS mean change of 4.47 $(p<0.001)$, ASES mean change of $40.6(p<0.001)$, and Constant score change of $26.1(p<0.001)$ (Tab. II). There were no post-operative complications experienced by any of the subjects; specifically, there were no post-operative infections or tendon ruptures 
Table I. Continuous variables expressed as the mean \pm standard deviation of the mean.

\begin{tabular}{llll}
\hline & Non-Injection $(\mathbf{n = 4 0})$ & Injection $(\mathbf{n = 9 2})$ & All $(\mathbf{n = 1 3 2})$ \\
\hline Age (yr) & $46.6 \pm 8.0$ & $48.9 \pm 10.7$ & $48.2 \pm 9.9$ \\
Male (\%) & 65 & 47 & 53 \\
Female (\%) & 35 & 53 & 47 \\
Mean Tear Length (cm) & $1.1 \pm 0.2$ & $1.0 \pm 0.2$ & $1.0 \pm 0.2$ \\
Tear Location: Articular (\%) & 42.5 & 38 & 39.4 \\
Tear Location: Bursal (\%) & 35 & 28.4 & 30.3 \\
Tear Location: Intratendinous (\%) & 22.5 & 33.6 & 30.3 \\
Traumatic Etiology (\%) & 45 & 45.7 & 45.5 \\
Mean Symptom Duration (m) & $15.1 \pm 23.2$ & $20.5 \pm 23.9$ & $18.9 \pm 23.7$ \\
Pre-operative Physical Therapy (\%) & 45 & 82 & 71 \\
\hline
\end{tabular}

Abbreviations: yr, years; cm, centimeters; m, months.

in patients who received a CSI prior to repair.

Of the 132 patients who satisfied the inclusion and exclusion criteria, 92 received a pre-operative CSI and 40 did not. Several reasons for foregoing an injection were mentioned in the records of the non-CSI group. The most common explanation was that a full complement of PT did not result in symptom relief, and the patient declined an injection in favor of pursuing surgery. Another common reason was that the patients' shoulder pain substantially interfered with either their work or normal recreation, and they preferred a surgical approach to their MRI-confirmed PTRCT. Other reasons included insufficient pain relief from a CSI in the opposite shoulder in the past, severity of symptoms and tendinosis seen on MRI, or in one case an allergy to a previous facet joint corticosteroid injection.

Initially, 40 patients who did not receive a pre-operative CSI were compared to 92 patients who did. Both groups independently showed significant improvements in all three outcome measures (Tab. II). The CSI group initially began at significantly worse levels in two of the three outcomes (VAS, $p=0.018$; ASES, $p=0.055$; Constant score, $p=0.032$ ). When the change in pre- and post-operative scores were directly com- pared with a paired t-test, the CSI group showed significantly greater improvement in all three metrics (Tab. III). Specifically, the CSI group improved 1.3 points more on VAS ( $p=0.005), 8.2$ points more on ASES $(p=0.019)$, and 7.0 points better on Constant score $(p=0.016)$. To examine whether the timing of pre-operative CSI influences post-operative outcomes, the injection group was further divided into three subgroups and their outcomes were compared. Initially, ANOVA showed significant variance between the subgroups in ASES $(p=0.037)$ and Constant score $(p=0.009)$ but not in VAS $(p=0.468)$. The subgroups were then compared with paired t-tests, and the results are presented in Table III. The 0-3 months CSI group showed significantly better improvements than the 3-6 months group in both ASES $(p=0.026)$ and Constant score $(p=0.004)$ and significantly greater improvements than the $>6$ months group in ASES $(p=0.023)$. In addition, the $0-3$ months subgroup showed significantly greater improvements in all three subgroups when compared to the non-injection group (VAS, $p=0.002$; ASES and Constant score $\mathrm{p}<0.001)$. There was no significant difference in any outcome measure between the 3-6 months and $>6$ months CSI subgroups.

Table II. Change in pre- and post-operative scores was analyzed with a paired student's t-test.

\begin{tabular}{lllllllllll}
\hline & \multicolumn{3}{c}{ VAS } & \multicolumn{3}{c}{ ASES } & \multicolumn{4}{c}{ Constant Score } \\
\hline Groups & $\#$ & Preop & Postop & p-value & Preop & Postop & p-value & Preop & Postop & p-value \\
Injection & 92 & 6.1 & 1.0 & $<0.001$ & 41.7 & 84.7 & $<0.001$ & 50.9 & 79.1 & 0.014 \\
Non-Injection & 40 & 5.0 & 1.3 & $<0.001$ & 48.5 & 83.4 & $<0.001$ & 57.1 & 78.4 & $<0.001$ \\
All & 132 & 5.8 & 1.1 & $<0.001$ & 43.7 & 84.3 & $<0.001$ & 52.8 & 78.9 & $<0.001$ \\
\hline
\end{tabular}

Abbreviations:Preop, pre-operative; Postop, post-operative; VAS, Visual Analog Scale; ASES, American Shoulder and Elbow Surgeon 
Table III. Statistical analysis was performed with a paired student's t-test.

\begin{tabular}{llllllllll}
\hline & \multicolumn{3}{c}{ VAS } & \multicolumn{3}{c}{ ASES } & \multicolumn{4}{c}{ Constant Score } \\
\hline Groups & Mean $\Delta$ & Mean $\Delta$ & p-value & Mean $\Delta$ & Mean $\Delta$ & p-value & Mean $\Delta$ & Mean $\Delta$ & $p$-value \\
Injection vs. Non-injection & 5.1 & 3.8 & 0.005 & 43.1 & 34.9 & 0.019 & 28.2 & 21.3 & 0.016 \\
0-3 months vs. Non-injection & 5.5 & 3.8 & 0.002 & 50.5 & 34.9 & $<0.001$ & 34.8 & 21.3 & $<0.001$ \\
0-3 moths vs. 3-6 months & 5.5 & 4.7 & 0.245 & 50.5 & 38.6 & 0.026 & 34.8 & 22.5 & 0.004 \\
0-3 months vs. >6 months & 5.5 & 5.0 & 0.410 & 50.5 & 41.1 & 0.023 & 34.8 & 28.1 & 0.346 \\
3-6 months vs. >6 months & 4.7 & 5.0 & 0.693 & 38.6 & 41.1 & 0.603 & 22.5 & 28.1 & 0.112 \\
\hline
\end{tabular}

Abbreviations: VAS, Visual Analog Scale; ASES, American Shoulder and Elbow Surgeon.

The multiple linear regression analysis found that undergoing a CSI 0-3 months prior to surgery was a primary predictor of functional outcomes in all three outcome measures (Tab. IV). The coefficients for the 0-3 months subgroup in VAS (2.295), ASES (18.782), and Constant score (14.559) are notably higher than all other variables and, moreover, at $p<0.001$. The other two CSI subgroups were also significant predictors for improvement in VAS but not ASES or Constant score. Undergoing surgery within 3 months of symptom onset was a significant predictor of improvement in Constant score (coefficient=19.484, $\mathrm{p}<0.001$ ) but not in ASES or VAS. Multiple CSI's were a prediction of poorer outcomes in all three metrics but not at a level of significance. All other variables included in the analysis were not significant predictors of outcomes $(p<0.05)$.

\section{Discussion}

The use of subacromial CSI's to treat RCT's and other pathologies of the shoulder joint is a long-established practice. Data supporting this practice, howev- er, are inconclusive. The results of several systematic reviews and meta-analyses over decades suggest there may be short-term symptomatic relief after CSI, but these results are often difficult to reproduce ${ }^{4-8}$. In addition, case reports from the past have suggested a correlation of CSI and tendon rupture ${ }^{19-21}$, which has been confirmed in multiple animal models ${ }^{6,9,10,22}$. Previous studies have also shown an increase in post-operative infection rates in patients who received pre- or peri-operative CSI's ${ }^{23-25}$, but recent systematic reviews and meta-analyses have challenged these claims ${ }^{11,13,26,28}$. Thus, not only there is a lack of consensus regarding CSI dosage, inter-injection interval, and type of corticosteroid to administer, but a body of research exists which challenges the safety of CSI's entirely. As a result, the use of CSI's to treat RCT's among clinicians is highly variable. Currently, the American Academy of Orthopaedic Surgeons (AAOS) guidelines are officially inconclusive regarding the use of corticosteroids to treat RCT's and defer the matter to physician discretion ${ }^{28}$. The purpose of this study was to test the hypothesis that a subacromial CSI prior to arthroscopic repair of a PTRCT would improve post-operative functional

Table IV. Multiple Linear Regression Analysis of the Temporal Relationship of Steroid Injection to Primary Outcomes.

\begin{tabular}{llll}
\hline & Coefficient & Standard Error & p-Value \\
\hline VAS Decrease & & & \\
0-3 months & 2.295 & 0.665 & $<0.001$ \\
3-6 months & 1.444 & 0.623 & 0.022 \\
$>6$ months & 1.316 & 0.657 & 0.047 \\
ASES Increase & & & \\
0-3 months & 18.782 & 5.145 & $<0.001$ \\
3-6 months & 6.732 & 4.821 & 0.165 \\
$>6$ months & 9.583 & 5.080 & 0.062 \\
& & & \\
Constant Increase & 14.559 & 3.790 & $<0.001$ \\
0-3 months & 3.698 & 3.551 & 0.300 \\
3-6 months & 7.314 & 3.742 & 0.053 \\
$>6$ months & & & \\
\hline
\end{tabular}

Abbreviations: VAS, Visual Analog Scale; ASES, American Shoulder and Elbow Surgeon. 
outcomes. To our knowledge, no study has directly tested this hypothesis. The concept of pre- or peri-operative injections of analgesics in order to improve post-operative outcomes is not foreign to orthopaedic surgery. The issue has been discussed at length with respect to surgeries of the lower extremity, and studies are currently being conducted to investigate the utility of adding corticosteroids to these local anesthetic cocktails. For instance, Stowers et al. conducted a systematic review of 14 randomized controlled trials comparing results of knee surgeries which included corticosteroids in the peri-operative local analgesic injection and those which did not ${ }^{15}$. The conclusion of this study was that the addition of a corticosteroid to the injection is safe and can reduce immediate postoperative pain, aid early knee range of motion recovery, and reduce narcotic consumption. In particular, no increased risk of infection was found with the addition of corticosteroid and, notably, none of the 1,271 patients included in the review experienced tendon rupture ${ }^{15}$. Other studies have shown similar results, namely that the addition of corticosteroid to peri-operative local injection results in improved pain levels and does not increase post-operative complications ${ }^{12,14,16,17,26,27}$. The significant improvement in post-operative functional outcomes following pre-operative CSI found in this study support similar conclusions, applied to PTRCT arthroscopic repair.

This study can be distinguished from the lower extremity studies mentioned above in two important ways: 1) our focus was on long-term outcomes at one year after surgery, not short-term results, and 2) the CSI's at issue were pre-operative and not peri-operative. In general, patients who received a CSI 0-3 months prior to surgery had significantly better outcomes one year after surgery. This phenomenon may suggest that the analgesic and anti-inflammatory effects of the corticosteroid allowed for earlier and increased post-operative rehabilitation of the repaired supraspinatus tendon. This then resulted in less pain and higher functionality 12 months after surgery when compared to patients who received a $\mathrm{CSI}$ at $>3$ months prior to surgery or none at all.

As noted above, there is current debate within the literature regarding the therapeutic duration of a CSI. One systematic review found CSI to be effective only at 2-4 weeks after administration ${ }^{8}$, while another found a variety of injected steroids to reduce pain and increase functionality up to 8 weeks ${ }^{7}$. The findings of this study suggest the duration of action may be longer than previously reported since patients who received a CSI up to 3 months prior to surgery still had greater functional improvement. Future studies are warranted to examine the ideal pre-operative interval for CSI's in order to maximize post-operative outcomes while ensuring patient safety.

There were several limitations to this study. While we have shown novel findings and good outcomes with pre-operative CSI's, this study was retrospective and bears all the limitations of such a design. In addition, concomitant procedures were included as part of this study and may have influenced outcomes. Our multivariate analysis, however, found no significant impact from these concomitant procedures. Post-operative outcomes were unfortunately limited to a one-year follow-up, which is a shorter period than generally would be preferred. Finally, the sample size presented here was relatively small with only 132 subjects. Future, larger studies are needed to continue investigation into this matter.

\section{Conclusion}

Until now, subacromial CSI's have been regarded solely as non-surgical treatment for RCT's with the goal of avoiding surgery by sufficiently relieving shoulder pain and inflammation. This study supports an additional use of CSI's as a pre-operative treatment to improve post-operative functional outcomes following arthroscopic repair of high-grade PTRCT's. Further investigation into the timing, dosage, and type of corticosteroid is needed and currently underway. In addition, the application of this pre-operative CSI protocol to full-thickness and massive RCT's is warranted.

\section{Conflict of interests}

The Authors declare they have no conflict of interests regarding the publication of this manuscript.

\section{References}

1. Yamamoto A, Takagishi K, Osawa T, et al. Prevalence and risk factors of a rotator cuff tear in the general population. J Shoulder Elbow Surg. 2010;19(1):116-120.

2. Ellman H. Diagnosis and treatment of incomplete rotator cuff tears. Clin Orthop Relat Res. 1990;(254):64-74.

3. Bhatia M, Singh B, Nicolaou N, Ravikumar KJ. Correlation between rotator cuff tears and repeated subacromial steroid injections: a case-controlled study. Ann R Coll Surg Engl. 2009 91(5):414-416.

4. Buchbinder R, Green S, Youd JM. Corticosteroid injections for shoulder pain. Cochrane Database Syst Rev. 2003;(1).

5. Contreras F, Brown HC, Marx RG. Predictors of success of corticosteroid injection for the management of rotator cuff disease. HSS J. 2013;9(1):2-5.

6. Dean BJ, Lostis E, Oakley T, Rombach I, Morrey ME, Carr AJ. The risks and benefits of glucocorticoid treatment for tendinopathy: a systematic review of the effects of local glucocorticoid on tendon. Semin Arthritis Rheum. 2014;43(4):570576.

7. Gaujoux-Viala C, Dougados M, Gossec L. Efficacy and safety of steroid injections for shoulder and elbow tendonitis: a metaanalysis of randomised controlled trials. Ann Rheum Dis. 2009;68(12):1843-1849.

8. Koester MC, Dunn WR, Kuhn JE, Spindler KP. The efficacy of subacromial corticosteroid injection in the treatment of rotator cuff disease: A systematic review. J Am Acad Orthop Surg. 2007;15(1):3-11.

9. Maman E, Yehuda C, Pritsch T, et al. Detrimental Effect of Repeated and Single Subacromial Corticosteroid Injections on 
the Intact and Injured Rotator Cuff: A Biomechanical and Imaging Study in Rats. Am J Sports Med. 2016 Jan:44:177-182.

10. Wei AS, Callaci JJ, Juknelis D, et al. The effect of corticosteroid on collagen expression in injured rotator cuff tendon. J Bone Joint Surg Am. 2006;88(6):1331-1338.

11. Christensen $C P$, Jacobs $C A$, Jennings $H R$. Effect of periarticular corticosteroid injections during total knee arthroplasty. A double-blind randomized trial. J Bone Joint Surg Am. 2009;91 (11):2550-2555.

12. Fajardo M, Collins J, Landa J, Adler E, Meere P, Di Cesare PE. Effect of a perioperative intra-articular injection on pain control and early range of motion following bilateral TKA. Orthopedics. 2011;34(5):354.

13. McMahon SE, LeRoux JA, Smith TO, Hing CB. Total joint arthroplasty following intra-articular steroid injection: a literature review. Acta Orthop Belg. 2013;79(6):672-679.

14. Salerno A, Hermann R. Efficacy and safety of steroid use for postoperative pain relief. Update and review of the medical literature. J Bone Joint Surg Am. 2006;88(6):1361-1372.

15. Stowers MDJ, Gao R, Penumarthy R, Munro JT. Periarticular use of Steroid for Knee Replacement Surgery. Ann Orthop Rheumatol. 2014;2(3):1028.

16. Yue DB, Wang BL, Liu KP, Guo WS. Efficacy of multimodal cocktail periarticular injection with or without steroid in tota knee arthroplasty. Chin Med J (Engl). 2013;126(20):38513855.

17. Zhao X, Qin J, Tan Y, Mohanan R, Hu D, Chen L. Efficacy of steroid addition to multimodal cocktail periarticular injection in total knee arthroplasty: a meta-analysis. J Orthop Surg Res. 2015;10:75.

18. Padulo J, Oliva F, Frizziero A, Maffulli N. Muscles, Ligaments and Tendons Journal - Basic principles and recommendations in clinical and field Science Research: 2016 Update. MLTJ.
2016;6(1):1-5.

19. Ismail AM, Balakrishnan R, Rajakumar MK, Lumpur K. Rupture of patellar ligament after steroid infiltration. Report of a case. J Bone Joint Surg Br. 1969;51:503-505.

20. Kleinman M, Gross AE. Achilles tendon rupture following steroid injection. Report of three cases. J Bone Joint Surg Am. 1983;65:1345-1347.

21. Stannard JP, Bucknell AL. Rupture of the triceps tendon associated with steroid injections. Am J Sports Med. 1993;21:482485.

22. Nakamura H, Gotoh M, Kanazawa T, et al. Effects of corticosteroids and hyaluronic acid on torn rotator cuff tendons in vitro and in rats. J Orthop Res. 2015;33(10):1523-1530.

23. Kaspar S, de V de Beer J. Infection in hip arthroplasty after previous injection of steroid. J Bone Joint Surg (Br). 2005;87 (4):454.

24. McIntosh AL, Hanssen AD, Wenger DE, et al. Recent intraarticular steroid injection may increase infection rates in primary THA. Clin Orthop Relat Res. 2006;451:50.

25. Papavasiliou AV, Isaac DL, Marimuthu R, et al. Infection in knee replacements after previous injection of intra-articular steroid. J Bone Joint Surg (Br). 2006;88(3):321.

26. Charalambous $C P$, Prodromidis AD, Kwaees TA. Do intra-articular steroid injections increase infection rates in subsequent arthroplasty? A systematic review and meta-analysis of comparative studies. J Arthroplasty. 2014;29(11):2175-2180.

27. Grumbine N, Dobrowolski C, Bernstein A. Retrospective evaluation of postoperative intralesional steroid injections on wound healing. J Foot Ankle Surg. 1998;37(2):135-144.

28. AAOS Guideline on the Optimizing the Management of Rotator Cuff Problems. http://www.aaos.org/research/guidelines/ RCPGuideline.asp. Published June 6, 2015. Accessed November 9, 2015. 